\title{
A CPG Synergy Model for Evaluation of Human Finger Tapping Movements
}

\author{
Keisuke Shima, Yasuhiro Tamura, Toshio Tsuji, Akihiko Kandori and Saburo Sakoda
}

\begin{abstract}
This paper proposes the CPG synergy model - a biomimetic rhythm generator model based on central pattern generators (CPGs) and muscle synergy theory to enable evaluation of rhythmic motions with non-stationary characteristics such as human finger tapping movements. The model consists of multiple CPGs to approximate the complex rhythmic movement of humans, and has the potential to allow evaluation of abnormal movements in patients with motor function impairments such as Parkinson's disease (PD).

To verify the validity of the proposed model, comparison experiments were conducted using model parameters (i.e., synergies, weight coefficients and time-shift parameters) extracted from finger tapping movements performed by individuals in a healthy subject group and a PD patient group. The results showed that the number of synergies, the second moment of synergy shapes and the coefficient of variation of maximum weight coefficients show significant differences for each subject group, and indicated that the model could be used to evaluate irregular rhythmic movements as well as regular ones.
\end{abstract}

\section{INTRODUCTION}

Parkinson's disease (PD) occurs due to the loss of nerve cells, which are responsible for producing dopamine in a portion of the midbrain known as the substantia nigra, and is a progressive nervous disease that causes motor function impairment resulting in symptoms such as rigidity, gait disorders and tremors.

Quantitative evaluation of voluntary/involuntary movements in PD patients has already been extensively researched through approaches such as the investigation of tremors by Salarian [1] and Gil et al. [2] and the examination of finger tapping movements by Konczak et al. [3], Shima et al. [4] and Yokoe et al. [5]. These studies sought to quantify the symptoms of PD patients by extracting the features of movements measured using various sensors, and investigated the motor function of patients and healthy subjects from the features of such movements. However, as voluntary human movements are generated by complex information processing in the central nervous system (CNS), the peripheral nervous system (PNS) and the musculoskeletal system (MSS), it is necessary to discuss the measured movement features and

This study was supported in part by a Grant-in-Aid for JSPS Fellows $(22 \cdot 8320)$ and Grant-in-Aid for Scientific Research (B) (21360118) from the Japan Society for the Promotion of Science.

K. Shima is with Graduate School of Biomedical Sciences, Hiroshima University, Hiroshima, 734-8551 Japan shima@bsys.hiroshima-u.ac.jp

Y. Tamura, and T. Tsuji are with Graduate School of Engineering, Hiroshima University, Higashi-hiroshima, 739-8527 Japan

A. Kandori is with Central Research Laboratory, Hitachi Ltd., Kokubunji, 185-8601 Japan

S. Sakoda is with Toneyama National Hospital, Toyonaka, 560-8552 Japan to develop a model-based evaluation tool in consideration of such information processing mechanisms.

The modeling of voluntary movements has two approaches: models for single-shot (discrete) movements such as reaching motions (referred to here as single movements) and those for periodic (continuous) movements such as walking (referred to here as rhythmic movements). For example, Flash et al. [6] proposed the minimum jerk model, and demonstrated the generation of a smooth hand trajectory with a bell-shaped velocity profile. Bizzi et al. [7] also proposed a method that can be employed to extract muscle synergies from time-series electromyograms (EMGs). However, it is not possible to generate and evaluate consecutive motions with these models due to their focus on single movements.

Rhythmic movements are generated by a CNS element called the central pattern generator (CPG) defined as a set of neural circuits that generate periodic motor commands for rhythmic movements such as locomotion. Calancie [9] and Dimitrijevic et al. [10] described the performance of rhythmic movements when electric stimulation was applied to spinal cord injury victims at constant intervals. Against this background, rhythmic movements such as walking have been modeled based on a CPG [11], [12]. It should be noted that CPG models cannot express non-stationary features of movements such as rapid changes in rhythm, amplitude and velocity even if the relevant structure can be determined appropriately.

The purpose of this study is to realize model-based evaluation of rhythmic movements with non-stationary characteristics in humans. To this end, we propose the CPG synergy model - a novel rhythm generator based on CPGs and muscle synergy theory. It consists of multiple CPGs to approximate complex rhythmic signals that change amplitude and velocity in each cycle. Modeling of rhythmic movements using the proposed model has the potential to allow evaluation of abnormal movements in PD patients.

\section{MODELING OF RHYTHMiC MOVEMENTS BASED ON A CPG SYNERGY}

\section{A. A CPG synergy model}

Rhythmic movements are known to be realized by periodic motor commands generated from CPGs in the spinal cord to the muscles, resulting in muscle activation. This paper considers finger tapping movements as an example of rhythmic movements (Fig. 1). Finger tapping movements, which have been widely applied in clinical environments to evaluate motor function, are periodic movements involving the opening and closing of the thumb and index finger. 

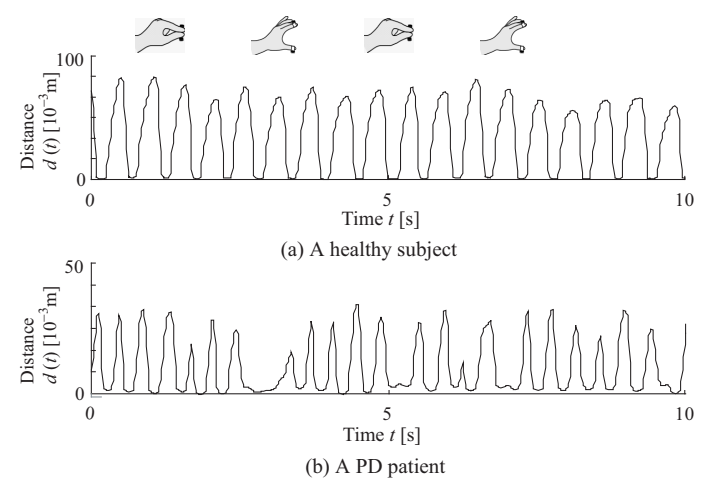

Fig. 1. Examples of finger tapping movements

Figure 1 plots the fingertip distances measured using a motor function evaluation system [4] for finger tapping movements proposed by Shima et al. The fingertip movement patterns of the healthy subject remain constant for each tap, while those of the PD patient show changes in features such as rhythm and amplitude for each tap, as pointed out in previous studies [1]-[4]. This example may suggest that the PD patient could not produce constant rhythmic patterns due to CPG disorders. Accordingly, to allow discussion of the relationships between the ability to generate motion and motor function impairment based on CPGs, a new model is required that enables the following:

- Expression of periodic movements

- Evaluation of rhythm patterns with rapid changes in features such as amplitude and velocity in each cycle

- Reconstruction of normal/abnormal rhythmic movements through dynamic adaptation of the parameters in the same model

Previous CPG models (such as those described in [13] and [14]) can predict periodic movements with stationary characteristics, but cannot produce rapid changes in rhythm. It is also difficult to reconstruct various movements using a single CPG model due to the complexity of setting the parameters involved.

This paper proposes a CPG synergy model that can be used for purposes such as generating complex rhythms combining multiple CPGs, which produce the building blocks of rhythm patterns by decomposing complex rhythmic signals into basic rhythm patterns. Figure 2 shows the proposed model, in which each CPG generates basic rhythm patterns that are then combined to produce various rhythmic movements using weight coefficients and time-shift parameters. By adapting these coefficients and parameters, the influences of each CPG on movements can be controlled, and rapid changes in amplitude and velocity for each cycle can be described. The signal measured from rhythmic movements is expressed

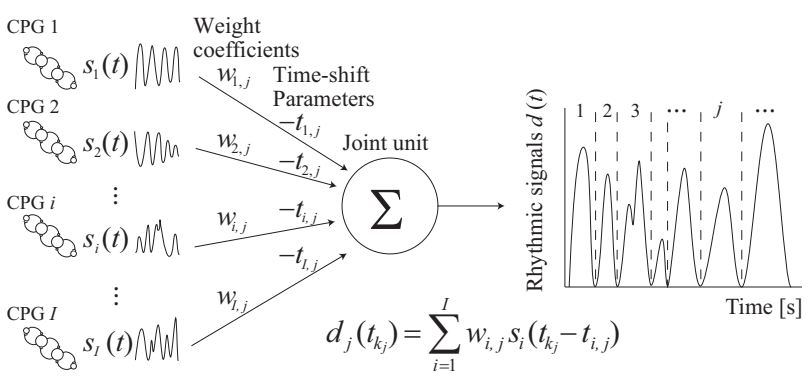

Fig. 2. Overview of the proposed CPG synergy model

as $d(t)$, which is defined as follows:

$$
\begin{aligned}
d(t) & =d_{j}\left(t_{k_{j}}\right) \\
t & =\left(\sum_{j^{\prime}=1}^{j} K_{j^{\prime}}+k_{j}-1\right) \Delta t, \\
t_{k} & =\left(k_{j}-1\right) \Delta t
\end{aligned}
$$

Here, $t=0, \Delta t, 2 \Delta t, \ldots,\left(\sum_{j=1}^{J} K_{j}-1\right) \Delta t$, and $d_{j}\left(t_{k_{j}}\right)$ is the value of $d(t)$ at $t_{k_{j}}$ in $j$ th cycle. $\Delta t$ is the sampling time, $j=1,2, \ldots, J$, and $k_{j}=0, \Delta t, 2 \Delta t, \ldots,\left(K_{j}-1\right) \Delta t . J$ is the number of cycles, $K_{j}$ represents the number of samples in the $j$ th period. The rhythmic patterns (synergies) generated by the $i$ th CPG are defined as $s_{i}$, and rhythm signals in the $j$ th cycle $d_{j}\left(t_{k_{j}}\right)$ are expressed as

$$
\begin{aligned}
d_{j}\left(t_{k_{j}}\right) & =\sum_{i=1}^{I} w_{i, j} s_{i}\left(t_{k_{j}}-t_{i, j}\right), \\
s_{i}((u-1) \Delta t) & =\left\{\begin{array}{ll}
S_{u}^{i} & \left(0 \leq(u-1) \Delta t<T_{\max }\right) \\
0 & \text { (otherwise) }
\end{array},\right.
\end{aligned}
$$

where $I$ describes the number of synergies, and $U$ is the number of samples in each synergy. $S_{u}^{i}>0$ indicates the value of the $u$ th sample in the $i$ th synergy, $T_{\max }=U \Delta t$ is the synergy duration time, and $w_{i, j}$ and $t_{i, j}$ are the amplitude and time-shift parameters for the $i$ th synergy (in the $j$ th cycle), respectively. In calculating the synergies $\boldsymbol{S}=\left[S_{u}^{1}, \ldots, S_{U}^{1}, S_{u}^{2}, \ldots, S_{U}^{2} \ldots, S_{u}^{I}, \ldots, S_{U}^{I}\right]^{\mathrm{T}}$ generated by each CPG, the weight coefficients $\boldsymbol{w}=\left[w_{i, j}\right] \in \Re^{I \times J}$ and time-shift parameters $t=\left[t_{i, j}\right] \in \Re^{I \times J}$ enable the expression of various rhythmic movements. It is therefore possible to investigate differences in the ability to generate motion between patients and healthy subjects based on the calculated parameters.

\section{B. Identification of the CPG synergy parameters}

The model parameters, which are synergies $S_{u}^{i}$, weight coefficients $w_{i, j}$ and time-shift parameters $t_{i, j}$, must be identified from the rhythmic signal $d_{j}\left(t_{k_{j}}\right)$. For parameter estimation, this study used the muscle synergy extraction algorithm proposed by Bizzi et al. [7], who described its ability to extract building blocks to simplify the construction of motor behavior (muscle synergy) from EMGs of frog's legs during kicking and jumping motion. It should also be noted that the measured EMGs could be reconstructed using 
a combination of the muscle synergies, weight coefficients and time-shift parameters extracted [8]. The identification procedure for the CPG synergy model is as follows:

i. To divide the signal of each cycle from $d(t)$, instants $t_{k_{j}}(=1,2, \ldots, J)$ when $d(t)<d_{t h}, \dot{d}(t)<0$ and $\dot{d}(t+$ $\Delta t) \geq 0$ are selected. Here, $J$ is the number of $t_{k_{j}} . d(t)$ is then normalized to make the maximum value equal to 1 and the minimum value equal to 0 . The normalized rhythmic signal $\mathbf{D}$ is expressed using $t_{k_{j}}$ as

$$
\begin{array}{r}
\mathbf{D}=\left[\hat{d}_{1}\left(t_{1}\right), \ldots \hat{d}_{1}\left(t_{K_{1}}\right), \hat{d}_{2}\left(t_{1}\right), \ldots, \hat{d}_{2}\left(t_{K_{2}}\right),\right. \\
\left.\ldots, \hat{d}_{J}\left(t_{1}\right), \ldots, \hat{d}_{J}\left(t_{K_{J}}\right)\right]^{\mathrm{T}}
\end{array}
$$

where $\dot{d}(t)$ is the temporal differentiation of $d(t)$.

ii. The synergy and weight coefficient elements, $S_{u}^{i}$ and $w_{k_{j}}$, are initialized using the following equations:

$$
\begin{aligned}
& S_{u}^{i}=\lambda \sin (2 \pi u \Delta t)+(1-\lambda) \mu(u \Delta t) \\
& w_{i, j}=\nu(i, j)
\end{aligned}
$$

where $\lambda(0<\lambda<1)$ is a constant set in advance, and $\mu(u \Delta t)$ and $\nu(i, j)$ are random values in the $[0,1]$ interval.

iii. Letting $\tilde{d}_{j}\left(t_{k_{j}}\right)=\hat{d}_{j}\left(t_{k_{j}}\right)$ and $r=1$, the time-shift parameter $t$ is found as outlined below.

iii-i. The maximum time delay is computed using the cross-correlation between $\tilde{d}_{j}\left(t_{k_{j}}\right)$ and $\left.s_{r}\left(t_{k_{j}}\right)\right)$, and is defined as the time-shift parameter $t_{r, j}$ of the $r$ th synergy. The model subtracts the synergy after weighting and time-shifting from the rhythmic signal $d_{j}\left(t_{k_{j}}\right)$ as $\tilde{d}_{j}\left(t_{k_{j}}\right)=\tilde{d}_{j}\left(t_{k_{j}}\right)-w_{r, j} s_{r}\left(t_{k_{j}}-t_{r, j}\right)$.

iii-ii. If $r<I$, it is set as $r=r+1$, and the process is repeated from iii-i. Meanwhile, if $r=I$, the process goes on to iv.

iv. The synergy and weight coefficients are updated using the multiplicative update rule [15] to minimize the evaluation function (i.e., root mean square errors, or RMS errors), expressed as

$$
E^{2}=\frac{1}{\sum_{j=1}^{J} K_{j}} \sum_{j=1}^{J} \sum_{k_{j}=1}^{K_{j}}\left(\hat{d}_{j}\left(t_{k_{j}}\right)-\sum_{i=1}^{I} w_{i, j} s_{i}\left(t_{k_{j}}-t_{i, j}\right)\right)^{2} .
$$

iv-i. The weight coefficients are updated using the following equation:

$$
w_{i, j}=w_{i, j}\left(\frac{\operatorname{trace}\left(\mathbf{D}_{j} \boldsymbol{S} \boldsymbol{\Theta}_{i}\left[t_{i, j}\right]\right)}{\operatorname{trace}\left(\mathbf{H}^{\mathrm{T}} \boldsymbol{S}^{\mathrm{T}} \boldsymbol{S} \boldsymbol{\Theta}_{i}\left[t_{i, j}\right]\right)}\right) .
$$

Here, $\boldsymbol{\Theta}_{i}$ is a matrix of the time-shift parameters, and is expressed as:

$$
\begin{aligned}
& {\left[\boldsymbol{\Theta}_{i}\left[\tau_{j}\right]\right]_{p q}=\delta[p-(i-1) j, q-j]} \\
& \mathbf{H}_{j}=\sum_{i}^{I} w_{i, j} \boldsymbol{\Theta}_{i}\left[t_{i, j}\right] \\
& \mathbf{H}=\left[\mathbf{H}_{1}, \mathbf{H}_{2}, \ldots, \mathbf{H}_{I}\right]
\end{aligned}
$$

where $\delta$ is the Kroneker delta.

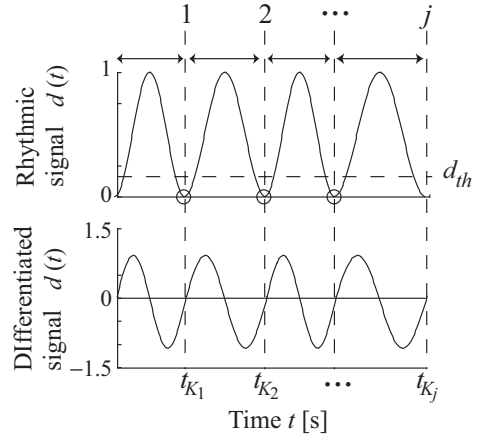

Fig. 3. Example of measured rhythmic signal

iv-ii. The synergies are updated using the following equation:

$$
\hat{\boldsymbol{S}}=\boldsymbol{S}\left(\frac{\mathrm{DH}^{\mathrm{T}}}{\boldsymbol{S H H}^{\mathrm{T}}}\right) .
$$

Here, the model calculates the determination coefficients $R^{2}$ and $\hat{R}^{2}$ between $\mathbf{D}$ and $\boldsymbol{S H}$, and between $\mathrm{D}$ and $\hat{\boldsymbol{S}} \mathbf{H}$, respectively.

v. Letting $\boldsymbol{S}=\hat{\boldsymbol{S}}$, the procedure is repeated from iv until $\left(\hat{R}^{2}-R^{2}\right)<R_{t h}^{2}$, where $R_{t h}^{2}$ is the threshold for termination of the algorithm.

Using extracted synergies $S_{u}^{i}$, weight coefficients $w_{i, j}$ and time-shift parameters $t_{i, j}$, various rhythmic movements can be expressed.

\section{EVALUATION OF FINGER TAPPING MOVEMENTS BASED ON A CPG SYNERGY MODEL}

The proposed model enables the extraction of synergies, weight coefficients and time-shift parameters for rhythmic movements, meaning that normality/abnormality in such movements can be evaluated based on the model parameters outlined above. This section discusses the ability to generate motion with healthy subjects and PD patients through evaluation of finger movements.

\section{A. Preliminary experiments}

To evaluate the ability to generate motion using the proposed model, an appropriate number of synergies must be decided to approximate the relevant movements. To this end, the relationships of estimated accuracy between the number of synergies and estimated movements were verified.

1) Methods: Synergy extraction experiments were conducted for finger tapping movement data recorded from two healthy female subjects (A: 24 years old; B: 26 years old). The subjects were asked to move their fingers in time with a metronome at a frequency of $3[\mathrm{~Hz}]$ for $15[\mathrm{~s}]$, and a magnetic sensor (UB-1; Hitachi Computer Peripherals Co., Ltd.) [16] was used to measure the finger movements. The sensor can output voltages corresponding to changes in the distance between the two coil-bearing fingertips, and these voltages are converted to fingertip distances based on non-linear calibration as proposed by Shima et al. [4]. 
The sampling frequency was $100[\mathrm{~Hz}]$, and one trial was conducted. The measured distances in a single finger tap (i.e., one opening and closing of the fingers) represent one cycle as rhythmic signals. Each tap was extracted from the velocities $\dot{d}(t)$ calculated from the fingertip distances $d(t)$ using differentiation filters. The parameters were set as $I=1$ to $7, T_{\max }=0.3$ (i.e., $U=30$ ), $\lambda=0.8$ and convergence condition $R_{t h}^{2}=0.001$.

2) Results and discussion: Figure 4 shows an example of the experimental results (Subject A; $I=4$ ) as follows: (a) measured fingertip distances and estimated signals from the proposal model; (b) extracted synergies; and (c) weight coefficients and time-shift parameters. The vertical and horizontal axes in Fig. 4 (a) show the amplitude of the fingertip distance and the given time $t$, the axes in (b) describe the values of four extracted synergies and the time, and the axes in (c) give the weight coefficients and time-shift parameters and the number of taps, respectively. In these results, the evaluation function $E^{2}$ and the determination coefficient between the measured and predicted distances are $0.047 \pm 0.006$ and $0.955 \pm 0.012$, respectively. The figure confirms that fingertip distances can be reconstructed using extracted synergies, weight coefficients and time-shift parameters, as the changes in the estimation waves are similar to those of the actual fingertip distances. In addition, all extracted synergies are bell-shaped, and the synergy with the maximum weight coefficient changes with each tap. These results indicate that the contribution rates of synergies change to express rhythmic movements with various amplitudes in each tap. Here, the weight coefficients and time-shift parameters in which the synergy contributed the most to the expression of each tap are defined as the maximum weight coefficients and maximum time-shift parameters.

Conversely, the extracted results of time-shift parameters (in Fig. 4(c)) show several large values of more than 0.3 [s] for the fourth synergy, indicating that this synergy is unnecessary for the estimation of movement. $J_{n}$ is the number of times when $t_{i, j}$ exceeds $0.33 \alpha$, and $\frac{J_{n}}{J}$ (where $J$ is the total number of taps, and $\alpha$ is a constant) is shown in Fig. 5. The vertical and horizontal axes show the number of synergies $I$, RMS errors and $\frac{J_{n}}{J}$ in $\alpha=0.9$. From the figure, it can be seen that $\frac{J_{n}}{J}$ became large in line with the increased number of synergies, while the evaluation functions did not change greatly. This result suggests that unnecessary synergies may be extracted if the number of synergies is too large for the expression of movements.

In this study, therefore, in order to determine the appropriate number of synergies, the number $N$ was increased in increments of one, and the maximum number of $N$ that satisfied $\frac{J_{n}}{J}<\beta$ was set as the appropriate number of synergies. Using this procedure, the number of synergies was set as $I=3$ for Subject A and $I=4$ for Subject $\mathrm{B}$ in the case of $\beta=0.1$. The evaluation function $E^{2}$ and the determination coefficient between the measured and predicted distances were $0.040 \pm 0.008$ and $0.957 \pm 0.017$, respectively. The results confirm that this procedure can be used to decide an appropriate number of synergies to

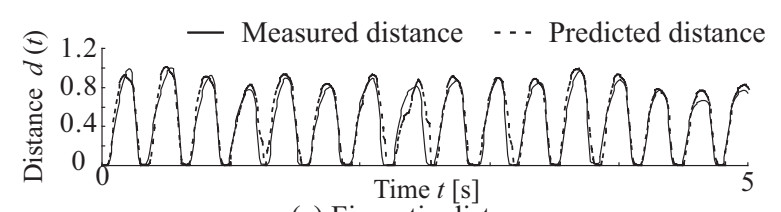

(a) Fingertip distance

$\rightarrow-$ Weight coefficients

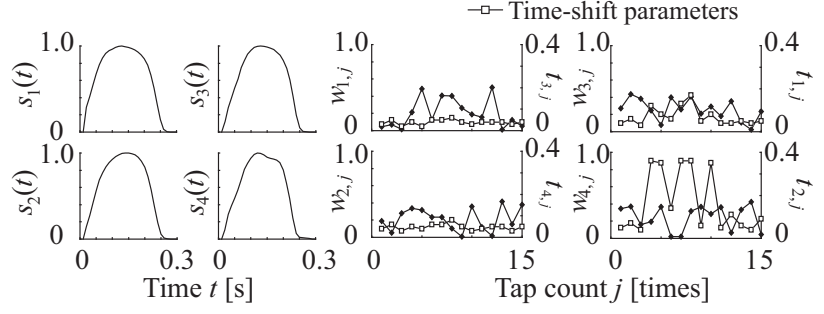

(b) Extracted synergies

(c) Weight coefficients and time-shift parameters

Fig. 4. Example of extracted synergies, weight coefficients and time-shift parameters in Subject A

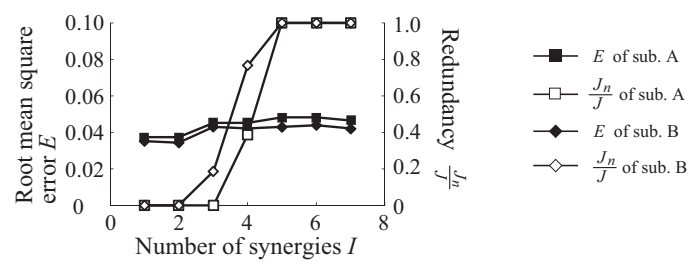

Fig. 5. Relationships between the number of synergies and estimation accuracies (RMS error and redundancy)

generate rhythmic movements for each subject.

\section{B. Evaluation of finger tapping movements for healthy sub- jects and PD patients}

1) Methods: Finger tapping data measured from healthy subjects and PD patients were verified using the proposed model as an example of evaluation of the ability to generate motion. The subjects were 30 healthy individuals (average age: $39.73 \pm 10.88$ years old, A - AD) and 3 PD patients (average age: $70 \pm 1.73$ years, $\mathrm{AE}$ - $\mathrm{AD}$ ), and were asked to move their fingers in time with a metronome at a frequency of $3[\mathrm{~Hz}]$ for $15[\mathrm{~s}]$. The parameters used in the proposed model were set as $T_{\max }=0.3, \lambda=0.8, R_{t h}^{2}=0.001, \alpha=$ 0.9 and $\beta=0.1$. To compare the ability to generate motion between healthy subjects and PD patients, four indices were computed: the number of synergies, the second moment of synergy shapes around the maximum value, the coefficient variation $(\mathrm{CV})$ of the maximum weight coefficients, and the $\mathrm{CV}$ of the maximum time-shift parameters.

2) Results: Examples of the results for healthy subjects and PD patients are shown in Figs. 6 and 7, respectively, which plot each value as follows: (a) finger tapping distance $d(t)$; (b) extracted synergies $s_{i}(t)$; (c) weight coefficients $w_{i, j}$ and time-shift parameters $t_{i, j}$; and (d), (e) maximum weight coefficients $w_{\max }$ and time-shift parameters $t_{\max }$ normalized by each mean value, respectively. The numbers of synergies found using the proposed procedure (as described 

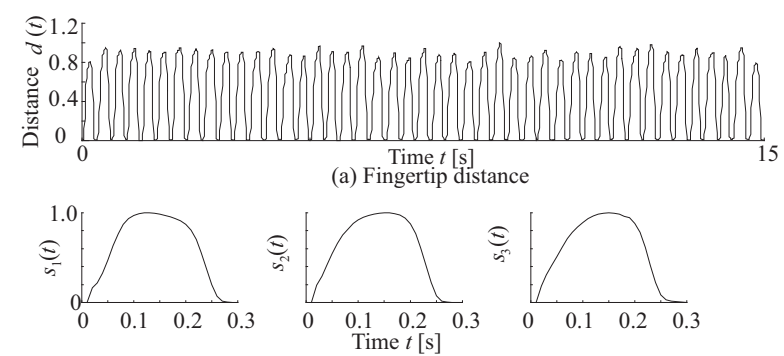

(b) Extracted synergies

- Weight coefficients $\quad-\square$ - Time-shift parameters

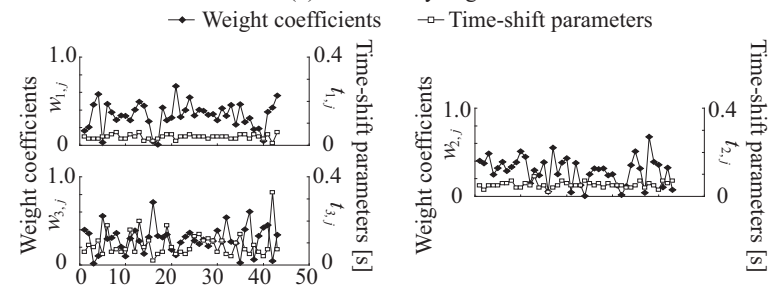

Tap count $j$ [times]

(c) Weight coefficients and time-shift parameters
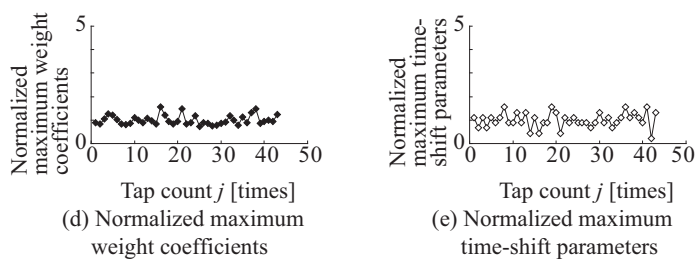

Fig. 6. An example of measured distance, synergies, weight coefficients and time-shift parameters (Subject C)

in Section 4.1.2) were 3 (Subject C - a healthy subject) and 8 (Subject AE - a PD patient). The approximation errors $E^{2}$ for each movement (i.e., the evaluation function of Eq. (9)) were 0.044 (Subject C) and 0.031 (Subject AE). The number of synergies, the second moment of synergy shapes around the maximum value, the coefficient variation of the maximum weight coefficients and the time-shift parameters are shown in Fig. 8, which plots the average values for all subjects. The average approximation errors for the healthy subject group and the PD patient group were $0.044 \pm 0.004$ and $0.037 \pm 0.005$, respectively, and the determination coefficients were $0.95 \pm 0.02$ and $0.96 \pm 0.01$, respectively. Since the numbers of synergies extracted from the healthy subject group and the PD patient group were $3.07 \pm 0.87$ and $8.00 \pm 1.00$, respectively, the number of synergies needed to express the movements of PD patients was higher than that for healthy subjects. The second moments of synergy shapes around the maximum value for each subject group were $0.044 \pm 0.014$ (healthy) and $0.016 \pm 0.006$ (PD), and the CVs of the maximum weight coefficients and time-shift parameters were $0.270 \pm 0.044$ and $0.511 \pm 0.016$ (healthy), and $0.410 \pm 0.117$ and $0.609 \pm 0.116$ (PD).

3) Discussion: Figure 6 (a) shows that the rhythms and amplitudes for the finger tapping distances of a healthy subject (Subject C) remained constant, while the movements of a PD patient (Subject AE) exhibited changes in features such as rhythm, amplitude and velocity with each tap. This
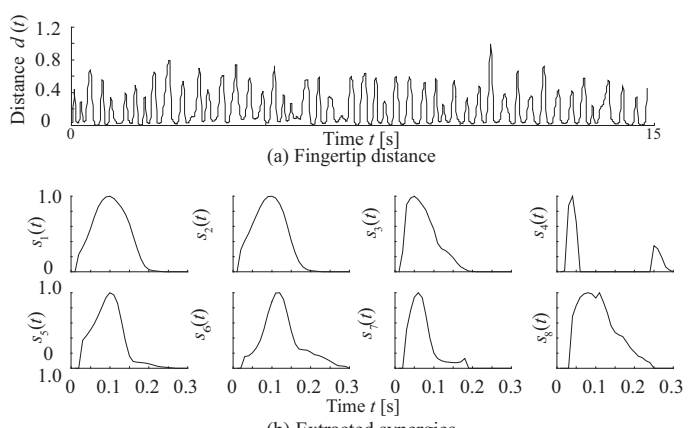

(b) Extracted synergies

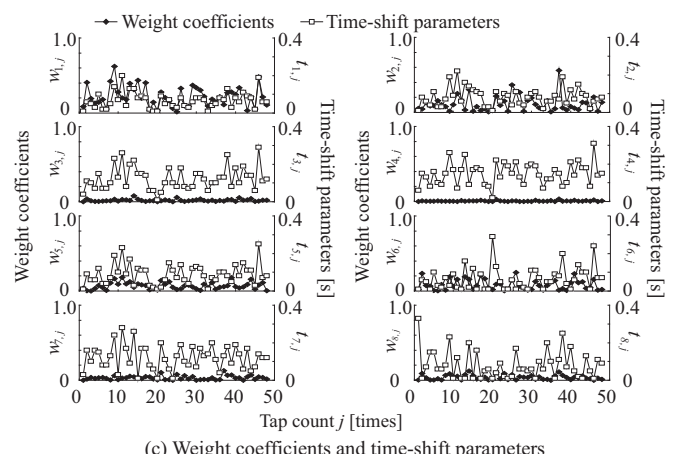

(c) Weight coefficients and time-shift parameters
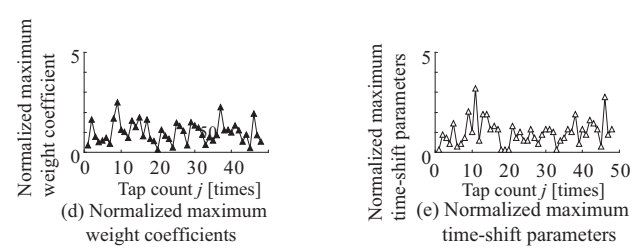

Fig. 7. An example of measured distance, synergies, weight coefficients and time-shift parameters (Subject AE)

symptom is recognized as typical of PD patient movements from previous studies [3]-[5] and in the UPDRS finger tapping score.

The average number of appropriate synergies for the PD patient group is larger than that for the healthy subject group. The shapes of the synergies extracted from PD patients are also relatively thin compared with those of healthy subjects. The average of the second moments (which represents the width of synergies) computed from PD patient group movements is smaller than that for healthy subjects (see Fig. 8 (b)). These results indicate that PD patients may have abnormal synergies with shapes different to those of healthy subjects, so many synergies may be needed to express their movements. In Figs. 6 and 7, the weight coefficients for both subjects become smaller when the number of synergies is large, which means that the contribution rate of synergies in finger tapping movements becomes small. Furthermore, the normalized maximum weight coefficients and time-shift parameters of the PD patient vary widely compared with those of the healthy subject, and the CVs of the maximum weight coefficients differ significantly $(p<0.01)$ for each subject group (Fig. 8 (c)). This result may indicate that the amplitudes of fingertip distance for PD patients gradually decrease due to loss of the adjustment function for weight 

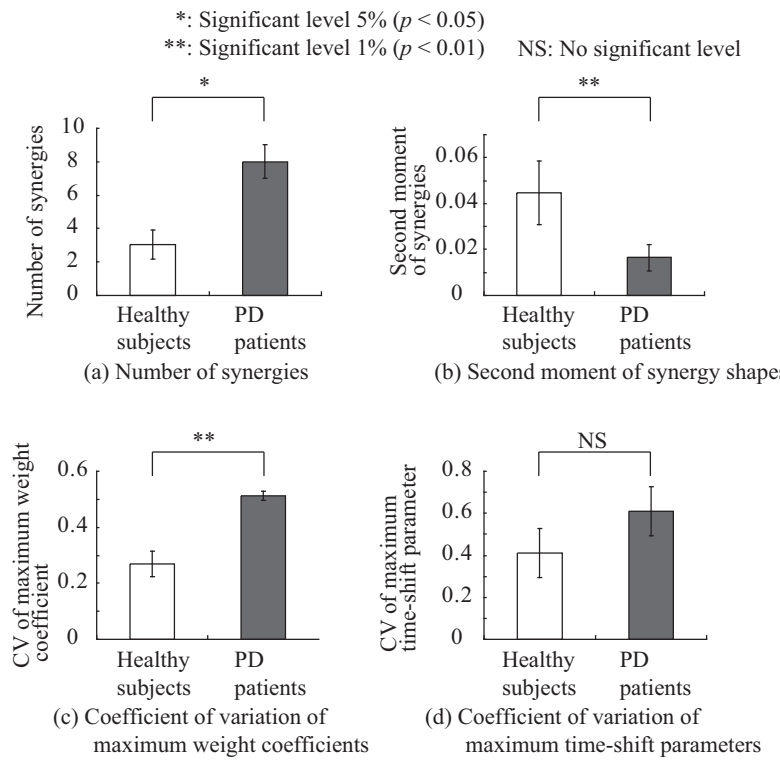

Fig. 8. Comparison results for healthy subjects and PD patients

coefficients in regard to synergies. However, the CVs of the maximum time-shift parameters in the PD patient group do not differ significantly (Fig. 8 (d)). Since the task set was to move the fingers in time with a metronome at $3[\mathrm{~Hz}]$, these variables did not differ between each subject group. This result leads us to the conclusion that the proposed model can evaluate the ability to generate motion, which may differ between healthy subjects and PD patients.

\section{CONCLUSION}

This paper proposes a novel CPG synergy model based on CPGs and muscle synergy theory to express complex human motions. The model can approximate irregular rhythmic movement and evaluate the ability to generate motion based on input parameters (i.e., synergies, weight coefficients and time-shift parameters) extracted from movements.

The results obtained in the comparison experiments for synergies, weight coefficients and time-shift parameters between the healthy subject group and the PD patient group are summarized below.

- More synergies may be needed to express movements in PD patients compared to those of healthy subjects (healthy subject group: $3.07 \pm 0.87$; PD patient group: $8.00 \pm 1.00)$.

- The shapes of synergies in PD patients are relatively thin compared with those of healthy subjects because the average second moments of synergy shapes around the maximum value are $0.044 \pm 0.014$ (healthy) and $0.016 \pm 0.006$ (PD).

- The CVs of the maximum weight coefficients (i.e., the weight coefficients of the synergies that contributed most to the expression of movements) for PD patients are significantly larger than those of healthy subjects (healthy: $0.270 \pm 0.044$; PD: $0.511 \pm 0.016$ ). tapping motion may differ between healthy subjects and PD patients.

In future research, we plan to investigate the approximation capability of the proposed model through comparison with that of the non-stationary AR model. Furthermore, the number of subjects will be increased to enable more detailed discussion of the ability to generate motion.

\section{REFERENCES}

[1] A. Salarian, H. Russmann, C. Wider, P.R. Burkhand, F.J.G. Vingerhoets, and K. Aminian: Quantification of Tremor and Bradykinesia in Parkinson's Disease Using a Novel Ambulatory Monitoring System, IEEE Transactions on Biomedical Engineering, Vol. 54, No. 2, 313/322, (2007)

[2] L.M. Gil, T.P. Nunes, F.H.S. Silva, A.C.D. Faria and P.L. Melo: Analysis of human tremor in patients with Parkinson Disease using entropy measures of signal complexity, 32nd Аnnual International Conference of the IEEE Engineering in Medicine and Biology Society, 2786/2789, (2010)

[3] J. Konczak, H. Ackermann, I. Hertrich, S. Spieker, J. Dichgans: Control of Repetitive Lip and Finger Movements in Parkinson's Disease, Movement Disorders, Vol. 12, No. 5, 665/676, (1997)

[4] K. Shima, T. Tsuji, E. Kan, A. Kandori, M. Yokoe, S. Sakoda: Measurement and Evaluation of Finger Tapping Movements Using Magnetic Sensors, Proceedings of the 30th Annual International Conference of the IEEE Engineering in Medicine and Biology Society, 5628/5631, (2008)

[5] M. Yokoe, R. Okuno, T. Hamasaki, Y. Kurachi, K. Akazawa and S. Sakoda: Opening velocity, a novel parameter, for finger tapping test in patients with Parkinson's disease, Parkinsonism and Related Disorders, Vol. 15, No. 6, 440/444, (2009)

[6] T. Flash and N. Hogan, The coordination of arm movements: An experimentally confirmed mathematical model, An experimentally confirmed mathematical model, Journal of Neuroscience, Vol. 5, 1688/1703, (1985)

[7] A. d'Avella, P. Saltiel and E. Bizzi: Combinations of muscle synergies in the construction of a natural motor behavior, nature neuroscience, Vol. 6, No. 3, 300/308, (2003)

[8] S.A. Overduln, A. d'Avella, J. Roh, E. Bizzi: Modularity of Muscle Synergy Recruitment in Primate Grasping, The Journal of Neuroscience, Vol. 28, No. 4, 880/892 (2008)

[9] B. Calancie, B. N. Shropshire, P. Jacobs, K. Willer, G. Zych and B. A. Green: Involuntary stepping after chronic spinal cord injury: evidence for a central rhythm generator for location in man, Brain, Vol. 117, 1143/1159, (1994)

[10] M. R. Dimitrijevic, Y. Gerasimenko, M. M. Pinter: Evidence for a spinal central pattern generator in humans, Ann N Y Acad Sci, 860, 360/376, (1998)

[11] G. Taga, Y. Yamaguchi and H. Shimizu,: Self-organized control of bipedal locomotion by neural oscillators in unpredictable environment, Biological Cybernetics, Vol. 65, 147/159, (1991)

[12] Y. Asai, T. Nomura, S. Sato, A. Tamaki, Y. Matsuo, I. Mizukura, K. Abe: A couple oscillator model of disordered interlimb coordination in patients with Parkinson's disease, Biological Cybernetics, Vol. 88, 152/162, (2003)

[13] H.R. Wilson and J.D. Cowan: Excitatory and inhibitory interactions in localize populations of model neurons, Biophysical Journal, Vol. 12, (1972)

[14] K. Matsuoka: Sustained Oscillatopns Generated by Mutually Inhibiting Neurons with Adaptation, Biological Cybernetics, Vol. 52, 267/376, (1985)

[15] D. D. Lee and H. S. Seung: Algorithms for non-negative matrix factorization, in Advances in Neural Information Processing Systems 13 (Proc. NIPS*2000), 556/562, (2000)

[16] A. Kandori, M. Yokoe, S. Sakoda, K. Abe, T. Miyashita, H. Oe, H. Naritomi, K. Ogata and K. Tsukada: Quantitative magnetic detection of finger movements in patients with Parkinson's disease, Neuroscience Research, Vol. 49, No. 2, 253/260, (2004)

These results indicate that the ability to generate finger 